\title{
Presentations associated with porphyrias in intensive care units
}

\author{
Doungporn Ruthirago MD, Parunyou Julayanont MD, Supannee Rassameehiran MD
}

\begin{abstract}
Porphyrias are a group of uncommon congenital metabolic diseases that are difficult to diagnose as they often present with nonspecific symptoms, mimicking other diseases. A significant number of patients with porphyrias have been admitted to intensive care units with acute abdominal pain, neuro-psychiatric symptoms, or hyponatremia. Due to the nonspecific symptoms, many patients have a delayed diagnosis. Moreover, some patients have received treatments that precipitate acute porphyric attacks, such as antibiotics or antiepileptics, causing worsening of symptoms. This article reviews the various clinical presentations and appropriate treatment of acute porphyrias in the ICU. Early diagnosis and appropriate management are important for preventing long-term disability and achieving good outcomes.
\end{abstract}

Key words- acute porphyria, pain, neuropathy, hyponatremia

\section{INTRODUCTION}

Porphyrias are a group of rare inherited metabolic disorders of heme biosynthesis. Congenital defects in the genes regulating the production of enzymes in each step of heme synthesis cause susceptibility to different types of porphyria. ${ }^{1}$ Porphyrias have a wide variety of clinical manifestations, but all of them are nonspecific and mimic diseases that are more common, making the diagnosis challenging and often delayed. Most patients who present with acute attacks of porphyrias require hospitalization, and many need intensive monitoring and treatment. Early diagnosis, appropriate management, and measures to prevent recurrent attacks are important for these patients.

\section{Classification}

Porphyrias were previously classified as hepatic and erythropoietic forms according to the site of the enzyme defect. Currently, they are

Corresponding author: Doungporn Ruthirago

Contact Information: Doungporn.ruthirago@ttuhsc.edu

DOI: $10.12746 /$ swrecc2016.0416.220 broadly classified as acute porphyrias and cutaneous porphyrias based on the clinical presentation. ${ }^{2,3}$

\section{Acute porphyrias}

The acute attack, which is a characteristic of acute porphyrias, often includes a triad: abdominal pain, neurological dysfunction, and psychiatric disturbances. Four of nine types are classified as acute porphyrias, specifically aminolevulinic acid dehydratase deficiency porphyria (ADP), acute intermittent porphyria (AIP), hereditary coproporphyria (HCP), and variegate porphyria (VP). The HCP and VP types can also present with cutaneous photosensitivity (Table 1). ${ }^{2,3}$

\section{Cutaneous porphyrias}

Porphyria cutanea tarda (PCT), erythropoietic protoporphyria (EPP), X-linked dominant protoporphyria (XLP), congenital erythropoietic porphyria (CEP), and hepatoerythropoietic porphyria (HEP) are classified in this group. These patients usually present with bullae, blisters, chronic cutaneous 
photosensitivity, skin fragility, and sometimes erosion or disfigurement of light-exposed areas (Table 1). ${ }^{3}$

\section{CLINICAL MANIFESTATIONS OF PORPHYRIAS IN INTENSIVE CARE UNITS AND MANAGEMENT}

Acute abdominal pain

Abdominal pain occurs in approximately $90 \%$ of patients. ${ }^{4}$ It usually presents as poorly localized pain with nausea, vomiting, abdominal distension, and constipation that is difficult to distinguish from other acute abdomen conditions. The mechanism of pain is neuropathic, so fever and leukocytosis rarely occur. However, porphyria patients often have extensive investigations and unnecessary surgeries for the abdominal pain.

Once porphyria is diagnosed, the pain can be controlled by acetaminophen, non-steroidal antiinflammatory drugs, or opiates as needed. Nausea and vomiting can be treated with promethazine, ondansetron, and other antiemetics. Constipation can be managed by laxatives, such as senna, lactulose, etc. ${ }^{3}$

\section{Acute neurological manifestations}

Acute porphyrias can present with different neurological symptoms that can be broadly classified into central nervous system, peripheral nervous system, and autonomic nervous system dysfunction.

Central nervous system manifestations include altered mental status, seizures, encephalopathy, and psychiatric disturbances, such as anxiety, depression, hallucinations, and paranoia. Seizures occur in 5-30\% of patients and can be triggered by hyponatremia from the syndrome of inappropriate antidiuretic hormone secretion (SIADH) or excessive gastrointestinal loss from vomiting. ${ }^{5}$ Hypomagnesemia can also precipitate seizures in these patients. Correction of water and electrolyte disturbances is important as well as appropriate antiepileptic medications. Primary antiepileptic agents, such as phenytoin, carbamazepine, and valproic acid, can precipitate porphyric attacks and increase symptoms. Benzodiazepines, such as diazepam (10 mg once only) and clonazepam, gabapentin, and magnesium sulfate can be used safely. ${ }^{3,6}$ Currently, there is inadequate information about the safety of levetiracetam in porphyria patients. In difficult-to-control seizures, propofol has been used effectively. It can also be used for sedation in the intensive care unit. $^{7}$

Neuropathy is reported in $20-68 \%$ of porphyria patients. The most typical pattern is axonal neuropathy with predominant motor involvement. $^{8}$ Sensory neuropathy is also reported but is less common. Cranial nerve and respiratory muscle paresis rarely occur but are signs of poor prognosis. ${ }^{3}$ Acute porphyria patients may require intubation for airway protection in case of encephalopathy or for respiratory support in case of respiratory muscle paresis. Patients with porphyric neuropathy usually require specific treatment with intravenous hemin and carbohydrate. Mechanical ventilation and intensive care monitoring are very important for patients with respiratory failure and seizures. Pain management for neuropathy, physical therapy, and speech therapy are also important.

Autonomic dysfunction is another common manifestation in acute porphyrias and is also a cause of mortality, such as sudden cardiac arrest. It can present with abdominal pain, tachycardia/bradycardia, hyper/hypotension, restlessness, sweating, and constipation. Patients with severe autonomic dysfunction require intensive care management. Severe hypertension and tachycardia can be managed with beta-blockers, such as propranolol, labetalol, and atenolol. ${ }^{3}$ However, blood pressures and heart rates in this group of patients sometimes fluctuate rapidly, causing significant difficulty in treatment. Some patients with severe hypotension and bradycardia may require vasopressors or inotropes. Urethral catheterization is needed in patients with sphincter and bladder dysfunction.

\section{Acute psychosis}

Psychiatric symptoms occur in $20-30 \%$ of patients during acute attacks. ${ }^{9}$ Acute psychiatric symptoms can be a central nervous system manifestation of porphyria or can be precipitated by other causes, such as severe hyponatremia and other toxic/metabolic encephalopathy. Monitoring and correction of these problems are 
important, especially in the intensive care setting. Benzodiazepines can be used for insomnia and anxiety. Antipsychotics, such as olanzapine and risperidone, are sometimes required for hallucinations and aggressive behavior. ${ }^{3}$

\section{Hyponatremia}

Hyponatremia occurs in approximately $30 \%$ of porphyria patients. ${ }^{4}$ The severity varies from mild to severe degree with serum sodium as low as $100-110 \mathrm{mg} / \mathrm{dl}$. Gastrointestinal sodium loss and SIADH from hypothalamic involvement are believed to be the main mechanisms. Maintaining fluid and electrolyte balance is very important and often difficult during porphyric attacks. The types of hyponatremia should be identified according to the patient's volume status and urine sodium level, followed by adequate replacement in case of hypovolemic hyponatremia or water restriction in case of SIADH. Patients with severe hyponatremia should receive $3 \%$ sodium chloride infusion (500 $\mathrm{mmol} / \mathrm{L}$ ) with close monitoring to prevent increases in serum sodium $>12 \mathrm{mmol} / \mathrm{L}$ per day. ${ }^{3}$

\section{COMMON PRECIPITATING FACTORS}

Environmental triggers that induce heme synthesis or disinhibit the enzyme in this pathway can precipitate porphyric attacks in patients with susceptible genes. ${ }^{10}$ These triggers are fever, infection, dehydration, prolonged fasting, alcohol, and several drugs, such as barbiturates, sulfonamides, female sex hormones, etc. (Table 2).

\section{DIAGNOSIS}

During acute attacks, the detection of elevated aminolevulinic acid (ALA) and porphobilinogen (PBG) in freshly voided urine is a good screening test. Measurement of total and individual porphyrins in serum, urine, and feces is helpful in both screening and differentiating the types of porphyrias. Quantitative measurement of enzyme activity in erythrocytes and lymphocytes support the diagnosis if the level is low. However, the gold standard test to confirm the diagnosis of porphyrias is DNA testing to identify specific mutations of each gene. ${ }^{1}$ Electrodiagnostic tests, namely electromyography and nerve conduction studies are useful in identifying the type of neuropathy and differentiating porphyrias from Guillain-Barré syndrome.

\section{TREATMENT}

Symptomatic treatments are mentioned earlier with each clinical presentation. Specific treatment of acute porphyrias should be started early in the course of disease to prevent severe nerve damage and incomplete recovery. Intravenous heme ( $4 \mathrm{mg} / \mathrm{kg} /$ day) for four days is the treatment of choice and should not be delayed in patients with possible porphyric attacks. Adequate carbohydrate intake and intravenous dextrose (300-500 g/day) help suppress disease activity and hasten recovery. ${ }^{1}$ Counseling patients to avoid precipitating triggers and counseling their family members to detect asymptomatic carriers are the most important steps to prevent future porphyric attacks.

\section{Conclusions}

Acute porphyrias can present with symptoms which occur frequently in intensive care unit patients; these include acute abdomen; central, peripheral nervous system, and autonomic dysfunction; psychiatric symptoms; and hyponatremia. Early diagnosis and appropriate treatment are very important during acute attacks to prevent morbidity and mortality from these diseases. A high index of suspicion is needed in patients who present with acute gastrointestinal and neuropsychiatric symptoms. Intensive care monitoring and treatment are crucial, especially for patients with seizures, severe autonomic dysfunction, and respiratory failure. 


\section{Table 1}

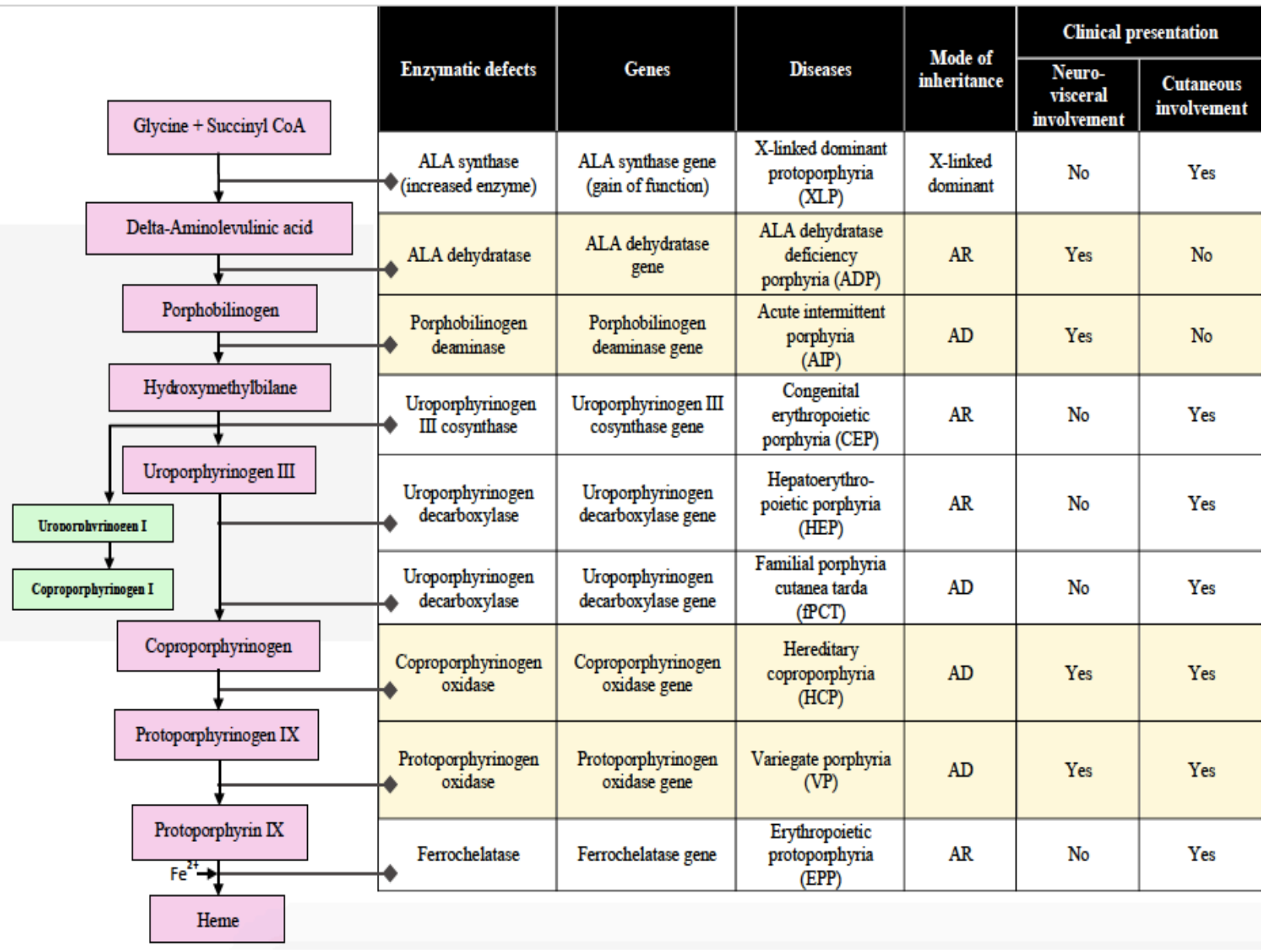

Table 2

Common drugs that can precipitate acute attack of porphyrias.

\begin{tabular}{lll} 
Barbiturates & Griseofulvin & Primidone \\
Carbamazepine & Methoxyflurane & Pyrazinamide \\
Carisoprodol & Methyldopa & Progestogens \\
Ergotamines & Oxcarbazine & Sulfonamides \\
Ethosuximide & Pentazocine & Thiopental \\
Etomidate & Phenytoin & Tiagabine \\
Felbamate & Phenobarbital & Topiramate \\
\hline
\end{tabular}




\section{REFERENCES}

1. The American Porphyria Foundation. Vol 2015. http://www.porphyriafoundation.com, Accessed October 24, 2015.

2. Balwani $\mathrm{M}$ and Desnick RJ. The porphyrias: advances in diagnosis and treatment. Blood 120: 4496-4504, 2012.

3. Puy H, Gouya L, and Deybach JC. Porphyrias. Lancet 375: 924-937, 2010.

4. Anderson KE, Bloomer JR, Bonkovsky HL, Kushner JP, Pierach CA, Pimstone NR, and Desnick RJ.

Recommendations for the diagnosis and treatment of the acute porphyrias. Ann Intern Med 142: 439-450, 2005.

5. Bylesjö I, Forsgren L, Lithner F, and Boman K.

Epidemiology and clinical characteristics of seizures in

patients with acute intermittent porphyria. Epilepsia 37: 230235, 1996.

6. Zadra M, Grandi R, Erli LC, Mirabile D, and Brambilla A. Treatment of seizures in acute intermittent porphyria: safety and efficacy of gabapentin. Seizure 7: 415-416, 1998.

7. Harrison JC and McAuley FT. Propofol for sedation in intensive care in a patient with an acute porphyric attack. Anaesthesia 47: 355-356, 1992.
8. Albers JW and Fink JK. Porphyric neuropathy. Muscle Nerve 30: 410-422, 2004.

9. Crimlisk HL. The little imitator--porphyria: a neuropsychiatric disorder. J Neurol Neurosurg Psychiatry 62: 319-328, 1997.

10. Meyer UA, Schuurmans MM, and Lindberg RL. Acute porphyrias: pathogenesis of neurological manifestations. Semin Liver Dis 18: 43-52, 1998.

\section{DETAILS}

1. Submitted-5/10/2016

2. Accepted-8/15/2016

3. Reviewers- Jongyeol Kim MD, Isham Huizar MD

4. Author affiliation- Doungporn Ruthirago MD and Parunyou Julayanont MD are residents in the Department of Neurology at Texas Tech University Health Sciences Center in Lubbock, TX. Supannee Rassameehiran MD was a resident in the Department of Internal Medicine at TTUHSC in Lubbock, TX.

5. Corresponding author- Doungporn Ruthirago at

Doungporn.ruthirago@ttuhsc.edu

6. Conflicts-none 